\title{
PRODUÇÃO DE UMA INTERFACE GRÁFICA (SOFTWARE ACADÊMICO) PARA SIMULAÇÃO DE UMA COLUNA DE DESTILAÇÃO
}

\author{
F. L. BEZERRA ${ }^{1}$, C.H. SODRÉ ${ }^{2}$ e A. MORAIS Jr ${ }^{3}$ \\ ${ }^{1,2}$ Universidade Federal de Alagoas, Centro de Tecnologia \\ ${ }^{3}$ Universidade Federal da Paraíba, Departamento de Engenharia Química \\ E-mail para contato: felipe_engquimica@hotmail.com
}

RESUMO: O conceito de simulação teórica para engenharia química está associado ao modelo matemático de determinado processo ou unidade, este modelo pode ser usado para gerar dados de um processo real utilizando equações matemáticas. A modelagem do processo deve ser iniciada na sua forma mais simples, aos poucos mudanças nas considerações iniciais vão sendo realizadas, de maneira que equações vão sendo adicionadas ou apenas modificadas no intuito de quando o modelo é simulado, os resultados produzidos tornam-se o mais próximo possível do processo real. Quando este estágio é alcançado, o modelo poderá ser utilizado para avaliar o comportamento do processo em diversas situações quando este é submetido a vários tipos de perturbações em diferentes variáveis. Com isso é possível ter: melhor entendimento do comportamento do processo; treinamento de operadores; estudo de otimização de processos; estudo do controle de processos, entre outros. Este tipo de análise não seria possível ser realizada no campo, devido à limitação das condições do processo. Neste sentido, partindo de um modelo computacional de uma coluna de destilação já funcionando em FORTRAN, será realizada a produção de uma interface gráfica no MATLAB para promover uma maior integração do programa simulador e o usuário. Neste caso, o usuário poderá realizar modificações nas condições de operação da coluna e observar os resultados sem a necessidade de ter conhecimento da linguagem de programação. 


\section{1 - INTRODUÇÃO}

A destilação é um dos processos mais utilizados na indústria química e petroquímica, por este motivo é uma operação amplamente estudada na engenharia química, sendo explorada nos mais diversos ramos, como na otimização de processos, materiais e equipamentos, instrumentação e controle. Um modelo deste processo já desenvolvido por Sodré (2001) vem sendo amplamente utilizado em atividades de pesquisa. Uma forma mais simples deste modelo também se encontra disponível para estudo da unidade de destilação.

No sentido de tornar este programa computacional mais dinâmico, surgiu a necessidade da criação de um sistema prático e de fácil acesso, que melhorasse a interação do usuário. Um sistema em que o usuário não tivesse acesso ao código fonte para fazer as mudanças necessárias, por exemplo, nos fluxos de variáveis, tipos de misturas e tipo de condensador para verificar como a separação é alterada mediante estas modificações. Muitas vezes, esta forma de intervenção do usuário pode alterar erradamente a estrutura do programa, além do que, decorar comandos e sintaxes de uma linguagem de programação de que não se tem domínio pode confundir o usuário. Nesta direção, o conceito de interface gráfica é importante para projetos de desenvolvimento de software, para os quais, a criação de janelas computacionais possibilita alcançar os objetivos finais, que neste caso é o conhecimento do processo de destilação. Neste artigo o modelo da coluna de destilação será referenciado como código fonte

\section{CONSIDERAÇÕES DO MODELO}

A coluna utilizada para a implementação do modelo e para a geração da interface é uma coluna seis pratos, com condensador total, refervedor parcial e transbordamento equimolar, a alimentação é feita no quinto estágio por definição, tendo a possibilidade de o usuário escolher em qual estágio será a alimentação.

A vazão de alimentação e a vazão de refluxo são subresfriadas, portanto modelo considera a condensação do vapor que ascende à coluna através dos balanços de energia.

O vapor gerado pelo refervedor é uma função da composição da mistura e da alimentação de vapor na sua serpentina, como mostra a eq.1.

$$
V_{r}=(0,0066) \cdot\left(V_{v}\right)+01426 \cdot 1000
$$

Onde $V_{v}$ depende da condição de equilíbrio líquido vapor, bem como da composição da mistura, e $V_{r}$ é a saída de vapor do reboiler. Tal equação foi concebida de forma experimental. 


\section{3 - FLUXOGRAMA DO MODELO}

O fluxograma inerente à coluna de destilação está apresentado na figura 1:

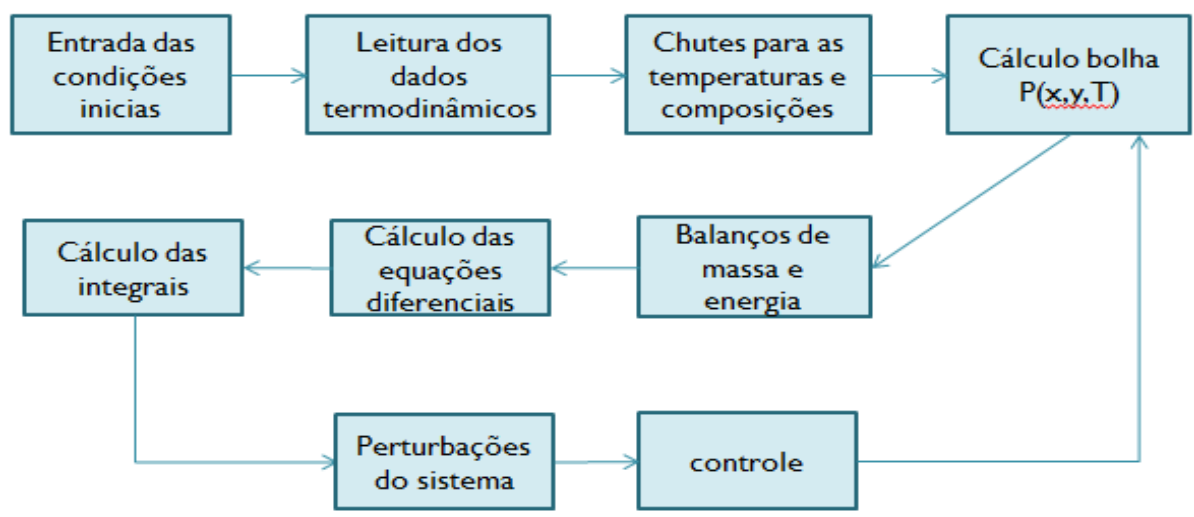

Figura 1: fluxograma do modelo

\subsection{Entrada das condições iniciais}

O modelo inicia com a entrada das condições iniciais, onde o usuário pode manipular diversas variáveis relacionadas com uma coluna de destilação como a vazão de entrada, razão de refluxo, vazão de valor, composição de entrada, a mistura a ser separada, entre outros. Com a alteração das condições de entrada a coluna irá responder de forma diferente.

\subsection{Leitura dos dados termodinâmicos}

Foi criado um banco de dados no EXCEL onde estão alocadas as constantes de Antoine, calores específicos, capacidades calorificas e calores latentes de cada composto. Atualmente o programa possibilita aproximadamente 60 misturas diferentes para serem simuladas no processo de destilação binária.

\subsection{Cálculo do ponto de bolha}

Após a leitura dos dados termodinâmicos, o programa realiza o cálculo do equilíbrio líquido-vapor, tendo como variáveis de entrada: a composição de líquida (x); a composição do vapor (y) e a pressão do sistema (P). A variável obtida pelo cálculo do ponto de bolha é a temperatura, onde essa nova temperatura é recalculada até que a condição de equilíbrio seja satisfeita. 


\subsection{Balanços de massa e energia}

Para o cálculo dos balanços de massa e componente foram utilizadas equações diferenciais que levam em consideração as vazões líquidas e de vapor para cada estágio bem como as composições. No balanço de energia considerou-se alimentação e vazão de refluxo sub-resfriadas, levando em consideração a condensação de parte do vapor que ascende a coluna.

\subsection{Cálculo das integrais}

As equações diferenciais foram resolvidas pelo método de Euler.

\subsection{Perturbações do sistema e controle}

A interface permite ao usuário que as perturbações no sistema sejam feitas em um instante de tempo predeterminado, o qual leva em consideração o tipo de mistura que está sendo utilizada. Logo após a etapa de integração, a variável que o usuário deseja alterar se atualiza para o valor da mudança, sendo possível e fácil a análise dinâmica do processo.

O programa também possui um sistema de controle, onde a variável controlada é a temperatura de topo e a manipulada é a vazão de refluxo, os parâmetros do controlador podem ser diretamente sintonizados pelo usuário na interface gráfica. Após esta etapa volta para o cálculo do bolha $\mathrm{P}(\mathrm{x}, \mathrm{y}, \mathrm{T})$, calculando uma nova temperatura e assim por diante.

\section{LAYOUT DA INTERFACE}

A figura 2 apresenta interface gráfico produzida no projeto, a mesma permite que o usuário simule dinamicamente o processo de destilação de forma prática e intuitiva. Cada painel da interface será explicado nesta seção. 


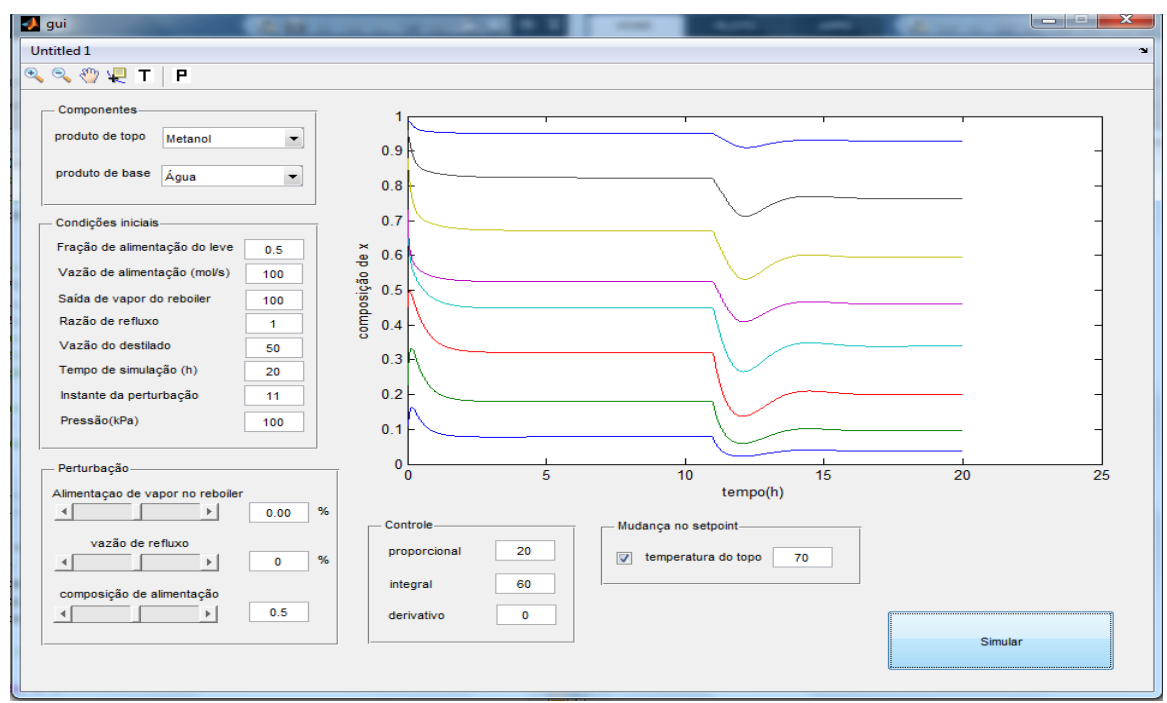

Figura 2: interface gráfica

\subsection{Componentes}

Aqui se tem a primeira condição que o usuário deve impor ao sistema, qual mistura ele quer que o programa simule. A figura 3 destaca este painel.

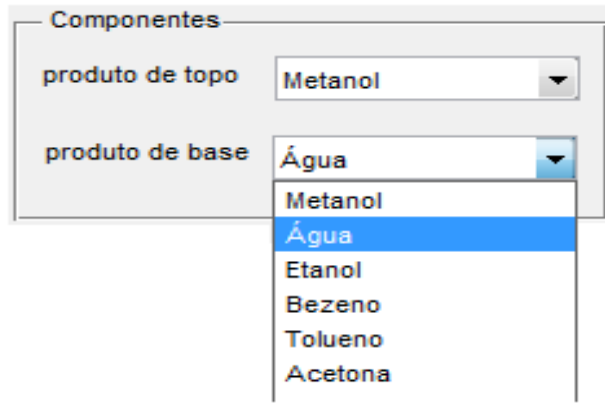

Figura 3: painel de componentes.

Como mostrado na fig.2 o usuário escolhe os produtos principais da mistura binária, tendo no produto de topo o composto mais volátil. $\mathrm{O}$ banco de dados referente às constante termodinâmicas estão situadas em um arquivo do Excel.

\subsection{Condições iniciais}

Neste painel é possível dar entrada em vários parâmetros para o processo de destilação. Ao inserir o valor desejado, o programa posiciona o mesmo no código do modelo. As variáveis que o simulador disponibiliza pra as condições iniciais são: fração de alimentação do composto mais volátil; vazão de alimentação; vazão de vapor que sai do reboiler e vai par a coluna; razão de refluxo; vazão de destilado; tempo de se deseja simular o processo; instante em de alguma perturbação ocorre; e a pressão. Este painel é mostrado na figura 3. 


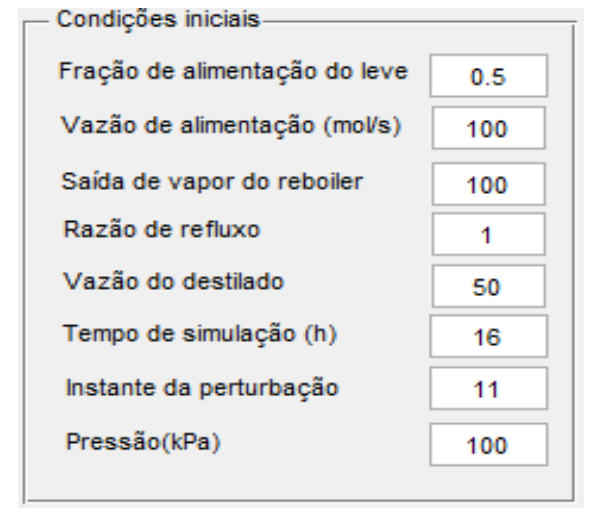

Figura 4: painel para as condições iniciais

\subsection{Perturbação}

Nesta opção é possível realizar distúrbios do sistema na vazão alimentação de vapor do reboiler, na vazão de refluxo e na composição de alimentação. As duas primeiras são em percentagem e a ultima em unidades de composição, onde se tem uma composição inicial estabelecida pelo usuário e a composição de perturbação.

Também se pode fazer mudanças no valor desejado da temperatura do estágio de topo, o que possibilita o estudo da malha de controle. A figura 5 mostra esse painel.

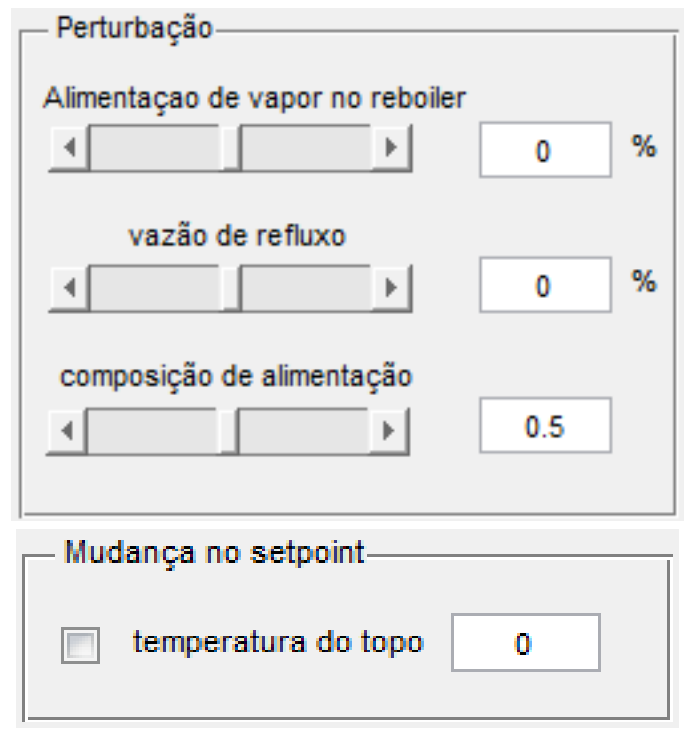

Figura 5: Painel de perturbações 


\subsection{Controle}

Para o estudo de controladores PID, implementou-se um sistema de forma que o usuário sintonize de forma simples o controlador de temperatura de topo (Smith e Corrípio).

O painel mostrado na figura 6 representa os ganhos: proporcional, integral derivativos. Onde, ao definir um valor para estes, o usuários está, automaticamente ativando o sistema de controle de acordo com os ganhos definidos, se o usuário deixar o valor nulo em algum ganho, este não irá atuar.

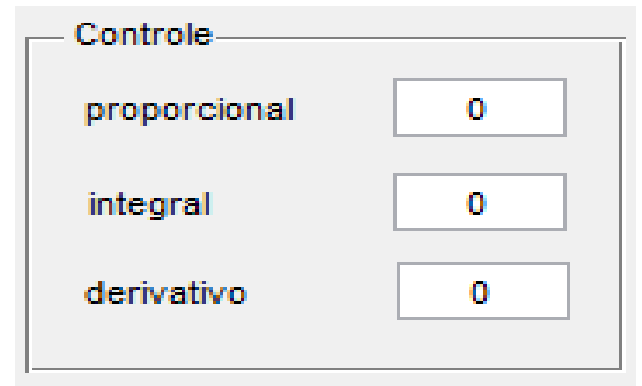

Figura 6: painel para o sistema de controle

\subsection{Saída de dados}

Após definir a condições iniciais, perturbações e tipo de controle, o usuário irá rodar o programa clicando no botão "Simular", ao fazer isso, o código gerado em MATLAB será compilado, realizando as devidas iterações, ao término do processo, o modelo irá gerar uma série de dados que serão salvos em EXCEL, além disso, a própria interface gera um diagrama da composição e/ou da temperatura ao longo do tempo, de todos os estágios como mostrado na figura 7 .

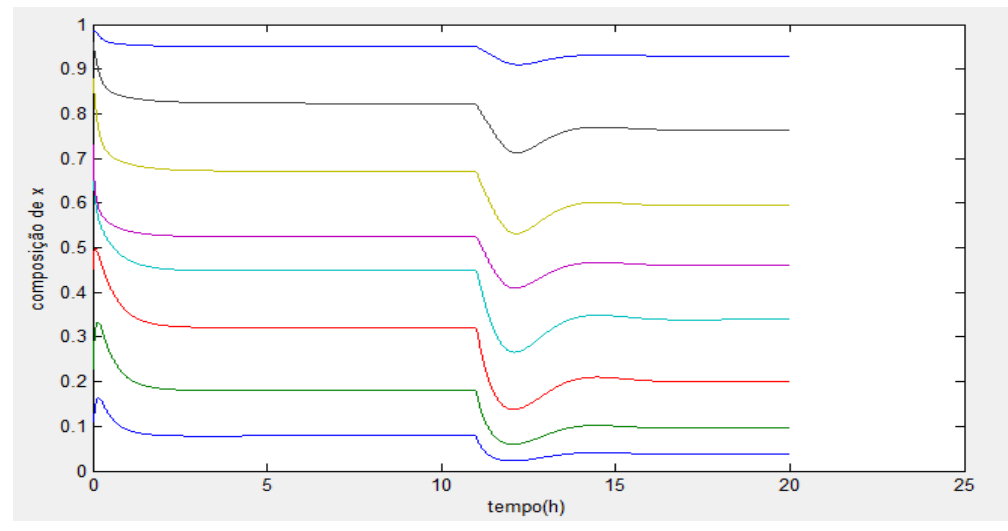

Figura 7: diagrama das composição de cada estágio ao longo do tempo. 
Na simulação, fez-se um degrau de $-10 \%$ na vazão de refluxo, num instante de tempo igual a $11 \mathrm{~h}$.

\section{CONSIDERAÇÕES FINAIS}

A aplicação de conceitos de simulação e controle de processos necessita de uma vasto conhecimento em linguagem de programação, tornado restrito o acesso à simulação de processos.

Neste artigo apresentou-se um simulador de uma coluna de destilação binária que facilita a interação homem-máquina através de uma interface gráfica.

Sua principal vantagem é que o usuário não necessita conhecer da linguagem de programação para simular o processo. Além disso, possui uma série de variáveis e de parâmetros que permitem o estudo dinâmico do processo, sendo assim uma ferramenta útil no desenvolvimento acadêmico.

\section{REFERÊNCIAS}

HANSELAMN, D; LITTLEFIELD, B. Matlab 6: Curso completo. Editor Pearson, $1^{\text {a }}$ edição.

SMITH, C; CORRÍPIO, A. Princípios e prática do controle automático de processos. $3^{\mathrm{a}}$ edição. 